\title{
Method for Determining Kinematic Parameters of the In Vivo Thumb Carpometacarpal Joint
}

\author{
Lillian Y. Chang*, Student Member, IEEE, and Nancy S. Pollard, Member, IEEE
}

\begin{abstract}
The mobility of the thumb carpometacarpal (CMC) joint is critical for functional grasping and manipulation tasks. We present an optimization technique for determining from surface marker measurements a subject-specific kinematic model of the in vivo CMC joint that is suitable for measuring mobility. Our anatomy-based cost metric scores a candidate joint model by the plausibility of the corresponding joint angle values and kinematic parameters rather than only the marker trajectory reconstruction error. The proposed method repeatably determines CMC joint models with anatomically-plausible directions for the two dominant rotational axes and a lesser range of motion (RoM) for the third rotational axis. We formulate a low-dimensional parameterization of the optimization domain by first solving for joint axis orientation variables that then constrain the search for the joint axis location variables. Individual CMC joint models were determined for 24 subjects. The directions of the flexion-extension (FE) axis and adduction-abduction (AA) axis deviated on average by $9^{\circ}$ and $22^{\circ}$, respectively, from the mean axis direction. The average RoM for FE, AA, and pronation-supination (PS) joint angles were $76^{\circ}, 43^{\circ}$, and $23^{\circ}$ for active $\mathrm{CMC}$ movement. The mean separation distance between the FE and $A A$ axes was $4.6 \mathrm{~mm}$, and the mean skew angle was $87^{\circ}$ from the positive flexion axis to the positive abduction axis.
\end{abstract}

Index Terms-Axes of rotation, optimization, subject-specific joint models, thumb mobility.

\section{INTRODUCTION}

$\mathbf{T}$ HE MOBILITY of the human thumb is a critical component of the hand's ability to grasp and manipulate objects. Subject-specific models can lead to more accurate evaluation of individual thumb motion and dynamic function. In this paper, we concentrate on the carpometacarpal (CMC) joint at the base of the thumb between the trapezium bone and metacarpal bone, which is responsible for the wide range of thumb circumduction and opposition [1], [2]. Determining appropriate, subject-specific models of this joint is important for evaluating individual thumb mobility with respect to the appropriate joint

Manuscript received July 31, 2007; revised December 19, 2007. This work was supported by the National Science Foundation under Grant CCF-0343161, Grant IIS-0326322, Grant ECS-0325383, Grant CNS-0423546, and Grant CCF0702443. The work of L. Y. Chang was supported by the National Science Foundation Graduate Research Fellowship. Asterisk indicates corresponding author.

${ }^{*}$ L. Y. Chang is with the Robotics Institute in the School of Computer Science, Carnegie Mellon University, Pittsburgh, PA 15213 USA (e-mail: lillianc@ri.cmu.edu).

N. S. Pollard is with the Robotics Institute and Computer Science Department in the School of Computer Science, Carnegie Mellon University, Pittsburgh, PA 15213 USA (e-mail: nsp@cs.cmu.edu).

Color versions of one or more of the figures in this paper are available online at http://ieeexplore.ieee.org.

Digital Object Identifier 10.1109/TBME.2008.919854 axes and creating customized hand models for virtual rehabilitation environments.

Previous work by Hollister et al. [3] located the anatomic rotational axes of the in vitro CMC joint, and further studies by Santos and Valero-Cuevas [4] simulated possible kinematic models based on distributions of joint parameters from cadaveric measurement. Cooney et al. [5] also determined the orientation of in vitro CMC axes from the ridges of the trapezium bone surface in cadaver hands, and these results reported with respect to the hand dorsum provide a single constant model of the axis orientations to subsequently measure in vivo CMC mobility. Noninvasive techniques proposed by Coert et al. [6] and Zhang et al. [7] evaluate thumb circumduction based on the observed motion of the thumb segments, without determining a kinematic model for the $\mathrm{CMC}$ axes. In contrast, Chèze et al. [8] and Cerveri et al. [9] have developed techniques for estimation of the in vivo CMC axes from isolated thumb movements such as circumduction and flexion-extension (FE). Our technique uses the entire joint range of motion (RoM) to determine an individual in vivo CMC joint model with two dominant rotational axes that are nonintersecting and nonorthogonal. The method accommodates any general surface marker protocol and can be used to measure joint RoM with respect to the subject-specific model fit to an individual's particular pattern of motion.

Several kinematic models have been used to describe the thumb CMC joint [3], [5], [10], [11]. The CMC joint motion is dominated by 2 degrees of freedom (DOF) of FE and adductionabduction (AA) and exhibits a lesser amount of pronationsupination (PS) [1], [5]. The simplest models [5], [10], [12] consider these axes as intersecting and orthogonal, as part of either a 2-DOF universal joint or a 3-DOF spherical joint. However, the anatomy of the interfacing bone surfaces of the trapezium and thumb metacarpal bones suggests a saddle joint model with two axes that are nonintersecting and nonorthogonal (skew) [1], [3], [13], and this has been incorporated in a five-virtual-link thumb model for simulation [4], [11].

We use a 3-DOF CMC joint model with nonintersecting FE and AA axes whose relative skew is determined by rotation about the PS axis. This model incorporates the complexity of the nonintersecting and nonorthogonal axes of the CMC saddle joint as in previous 2-DOF models [3], [4], [11], while also allowing measurement of the PS rotation as the third DOF. Our approach optimizes an individual CMC joint model over a lowdimensional parameter space by decomposing the joint model into the two parts of joint axis orientation and joint axis location. The cost metric for the optimization scores anatomical characteristics of the CMC joint motion rather than only evaluating the marker trajectory reconstruction error as used previously for 


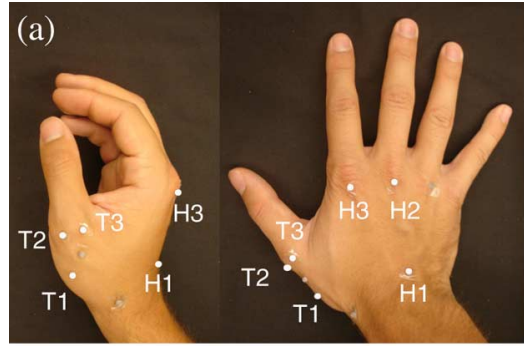

(b)

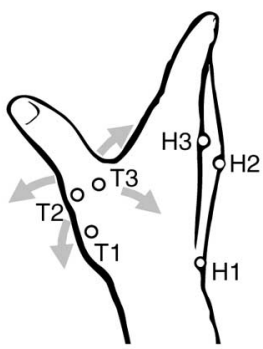

Fig. 1. (a) Marker protocol for the right hand used to measure the motion of the CMC joint in the experimental validation. Markers $\mathrm{H} 1, \mathrm{H} 2$, and $\mathrm{H} 3$ define the TCS for the hand dorsum, and markers T1, T2, and T3 define the TCS of the thumb metacarpal segment. A symmetric placement of markers was used for the left hand. The same marker protocol was used for all subjects to facilitate comparisons of the CMC axes locations, but the method can accommodate an arbitrary choice of the hand dorsum TCS and thumb metacarpal TCS. (b) Active RoM was measured while the subject exercised the CMC joint without any contact to the thumb.

similar joint models [14]-[16]. We find that to achieve subjectspecific models of the CMC joint that also have anatomicallymeaningful joint properties, minimizing the reconstruction error is insufficient because it leads to inconsistent estimates of axis directions and large RoM for the nondominant DOF. Instead, optimizing our proposed metric determines a joint model that has anatomically-meaningful joint properties such as the relative RoM between the DOFs and the proximal-distal relative location of the axes.

\section{METHOD}

\section{A. Experimental Protocol}

The study involved a total of 24 able-bodied individuals, consisting of 12 males (11 right-hand dominant, 1 left-hand dominant) and 12 females (11 right-hand dominant, 1 left-hand dominant) subjects, aged $26 \pm 3.2$ years (mean \pm standard deviation). A Vicon camera system tracked the positions of reflective surface markers attached to the hand dorsum and thumb metacarpal segment, which defined the hand technical coordinate system (TCS) and thumb metacarpal TCS [Fig. 1(a)]. Markers H1, H2, and H3 define the hand dorsum TCS and are attached, respectively, to the proximal end of the third metacarpal, the distal end of the third metacarpal, and the distal end of the second metacarpal. Markers T1, T2, and T3 define the thumb metacarpal TCS and are attached, respectively, to the proximal end of the first metacarpal on the radial side, the distal end of the first metacarpal on the radial side, and the distal end of the first metacarpal on the ulnar side. The marker locations were chosen to be spread out over the metacarpals of the hand dorsum and thumb to avoid, to the extent possible, large changes in the TCS orientation due to skin motion over the bone.

Subjects were seated at a table with the arm comfortably extended and the ulnar part of the hand in contact with the table surface for the data acquisition session [Fig. 1(b)]. The calibration movement for sampling the full space of CMC joint configurations consisted of circumduction, a star pattern, AA, and FE motions (Fig. 2). Subjects were directed to avoid motion of the other joints of the thumb, palm, and fingers, although

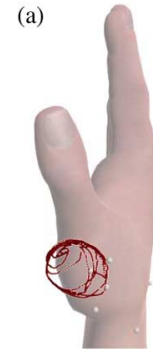

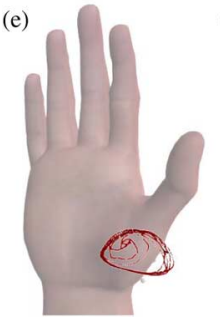

(b)

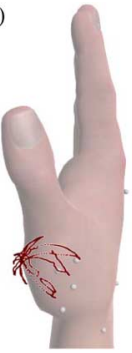

(f)

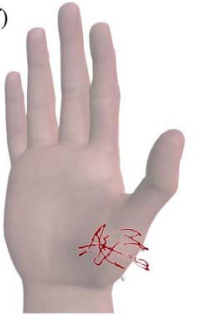

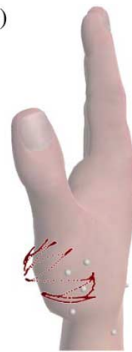

(g)

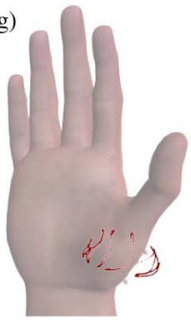

(d)

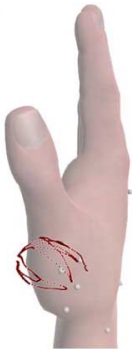

(h)

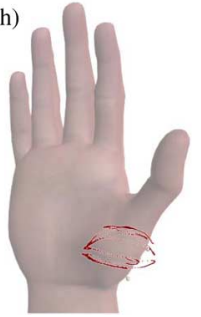

Fig. 2. Example of the calibration motion pattern measured from one subject. The trajectory of the T2 marker for the four portions of the motion pattern segment are shown for both radial and palmar views of the hand. (a) and (e) Clockwise and counterclockwise circumduction. (b) and (f) Star pattern with arcs across the joint RoM in multiple directions. (c) and (g) Zig-zag pattern with high-frequency AA while gradually changing the FE angle. (d) and (h) Zig-zag pattern with high-frequency FE while gradually changing the AA angle.

these joints were not mechanically constrained in order to encourage natural motion of the CMC joint. Subjects performed the calibration movement at a self-selected speed to exercise the active RoM of the CMC joint without any external contact to the thumb. Two repetitions of the movement pattern were recorded. The experiment was completed for both the right hand and left hand of each subject.

The recorded marker trajectories were lightly conditioned before use as input data as follows. Segments of static poses were manually clipped from the beginning and end of each motion sequence. In addition, any time samples with occluded markers were discarded. The resultant data sequence for one repetition of the calibration movement had on average 4500 time samples. For each of these remaining time samples, we computed the measured rigid transform $A_{m}$ of the thumb metacarpal TCS with respect to the hand dorsum TCS [Fig. 3(a)], which consists of the relative orientation matrix $R_{m}$ between the TCS axes and the position $p_{m}$ of the metacarpal TCS origin in the hand TCS frame. In addition, since the selection of the origin marker for the metacarpal TCS is arbitrary, our available data include the positions $\left(p_{m 1}, p_{m 2}, p_{m 3}\right)$ of all three metacarpal markers in the hand TCS frame.

\section{B. Problem Statement}

Given the sequence of measured relative TCS transforms $A_{m}$ and marker positions $\left(p_{m 1}, p_{m 2}, p_{m 3}\right)$ from the data acquisition, we wish to determine the joint model that describes the orientation and location of the functional $\mathrm{CMC}$ rotational axes. In our model of the CMC joint [Fig. 3(b)], the joint angles $\theta_{1}, \theta_{2}$, and $\theta_{3}$ denote three sequential rotations about the FE axis $z_{h}$, the $\mathrm{PS}$ axis, and the AA axis $z_{t}$, respectively. The FE axis $z_{h}$ is the 

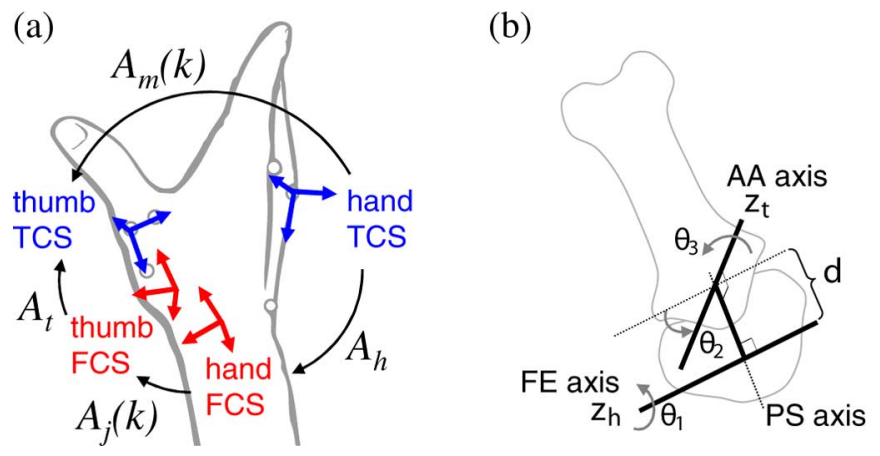

Fig. 3. (a) TCSs and FCSs that define the hand dorsum and thumb metacarpal segments adjacent to the CMC joint. The transform $A_{m}(k)$ describing the measured configuration of the $\mathrm{CMC}$ joint at time instance $k$ is the composition of the change-of-coordinate transform $A_{h}$, the CMC configuration expressed relative to functional coordinate systems $A_{j}(k)$, and the change-of-coordinate transform $A_{t}$. (b) Model of the three rotational axes of the CMC saddle joint. The FE axis $z_{h}$ is the $z$-axis of the hand dorsum FCS and is located through the trapezium bone. The AA axis $z_{t}$ is the $z$-axis of the thumb metacarpal bone FCS. The FE and AA axes are separated by distance $d$ along their mutual perpendicular that is the PS axis. The skew between the FE and AA axes is defined by the rotation angle $\theta_{2}$ about the PS axis.

$z$-axis of the hand dorsum functional coordinate system (FCS), and the AA axis $z_{t}$ is the $z$-axis of the thumb metacarpal FCS. The FE and AA axes are separated by a distance $d$ along the PS axis, and the value of the PS rotation angle determines the skew between the FE axis and AA axis. For a complete description of the joint model, we need to solve for the hand dorsum FCS and thumb metacarpal FCS [Fig. 3(a)] that express the CMC movement in terms of functional FE, AA, and PS joint angles. We also need to solve for the constant separation $d$ between the dominant FE and AA axes.

\section{Optimization Cost Metric}

To determine the joint model for an individual subject, we will use an optimization approach to select an appropriate set of hand FCS, thumb FCS, and separation $d$ that result in rotational DOFs $\theta_{1}, \theta_{2}$, and $\theta_{3}$ that both reconstruct the joint orientation and correspond to anatomically-meaningful FE, PS, and AA joint angles. We design our optimization cost metric for scoring candidate models as a combination of three cost components based on anatomical knowledge of the thumb CMC joint. The first aspect of the CMC joint motion is that it is dominated by $\mathrm{FE}$ and AA rotation with a limited amount of PS rotation, as reflected by the 2-DOF models used in previous work [4], [11]. Thus, the functional joint axes should correspond to joint variable sequences with small variation of $\theta_{2}$ values. The second aspect is that the FE RoM is generally larger than the AA RoM for unimpaired CMC joints [5], [6]. Finally, the FE axis fixed in the hand frame is proximal to the AA axis fixed in the thumb metacarpal frame due to the saddle joint geometry between the metacarpal and trapezium bones [3], [4], [11], [13]. These three anatomical aspects of the CMC joint kinematics are incorporated into an overall cost metric that is minimized to solve for meaningful joint axes. All three components are used simultaneously since a single component alone is not sufficient to satisfy all three objectives:

$$
f=f_{\mathrm{PS}}+f_{\mathrm{RoM}}+f_{d} .
$$

The component $f_{\mathrm{PS}}$ reflects the property that the observed PS angle values should have small variation as the nondominant rotational freedom. We motivate this cost metric component by a probabilistic model that assumes that the PS angle values are normally distributed with mean value $\mu_{2}$ and variance $\sigma_{2}^{2}$. To maximize the probability of observing a sequence of $\theta_{2}$ values, we can equivalently minimize the corresponding negative log likelihood function of the modeled normal distribution, normalized by the sequence length $N$ [17]. This is used as one component of the cost metric

$$
f_{\mathrm{PS}}=\frac{\sum_{k=1}^{N}\left(\theta_{2}(k)-\mu_{2}\right)^{2}}{2 N \sigma_{2}^{2}}
$$

where $k$ indicates a single time sample in the sequence.

Since the absolute value of the angle is irrelevant for describing the overall amount of PS rotation, there is no prior set for $\mu_{2}$ and we instead use the sample mean of the joint angle sequence

$$
\mu_{2}=\frac{1}{N} \sum_{k=1}^{N} \theta_{2}(k)
$$

The resultant form of (2) can be interpreted as the sample variance of the $\theta_{2}$ values, scaled by a weighting factor $2 \sigma_{2}^{2}$.

Next, the minimizing cost metric favors joint axis orientations that are aligned such that the FE RoM is maximized relative to the AA RoM

$$
f_{\mathrm{RoM}}=\frac{\operatorname{range}\left(\theta_{3}\right)}{\operatorname{range}\left(\theta_{1}\right)}
$$

where

$$
\operatorname{range}\left(\theta_{i}\right)=\max _{k \in(1, N)}\left(\theta_{i}(k)\right)-\min _{k \in(1, N)}\left(\theta_{i}(k)\right) .
$$

The final cost component measures how meaningful the estimated constant separation $d$ is with respect to an assumed normal distribution with mean $\mu_{d}$ and variance $\sigma_{d}^{2}$. As earlier, maximizing the probability of the estimated separation $d$ is equivalent to minimizing the negative $\log$ likelihood of $d$ :

$$
f_{d}=\frac{\left(d-\mu_{d}\right)^{2}}{2 \sigma_{d}^{2}}
$$

In this case, $\mu_{d}$ and $\sigma_{d}$ provide intuitive weighting parameters that represent our prior belief in the possible values for anatomically-plausible separation distances. Selecting $\mu_{d} \geq 0$ represents the choice to favor $\mathrm{CMC}$ joint models where the $\mathrm{FE}$ axis is proximal to the AA axis, to be consistent with the CMC bone anatomy.

The overall optimization cost metric

$$
f=\frac{\sum_{k=1}^{N}\left(\theta_{2}(k)-\mu_{2}\right)^{2}}{2 N \sigma_{2}^{2}}+\frac{\operatorname{range}\left(\theta_{3}\right)}{\operatorname{range}\left(\theta_{1}\right)}+\frac{\left(d-\mu_{d}\right)^{2}}{2 \sigma_{d}^{2}}
$$

evaluates how well a candidate joint model corresponds to a meaningful decomposition of joint angles with an appropriate separation distance between the FE and AA axes. The three tuning parameters $\left(\sigma_{2}, \mu_{d}, \sigma_{d}\right)$ are used to adjust the relative 


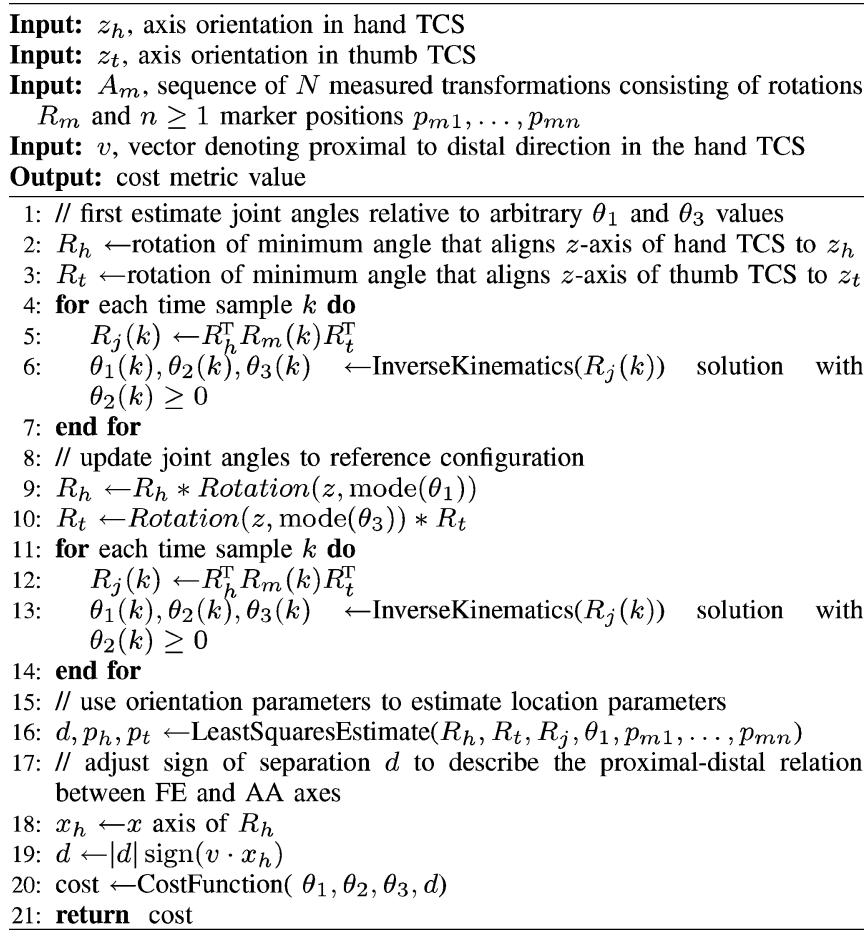

Fig. 4. Pseudocode for the evaluation of a 4-DOF solution family $\left(z_{h}, z_{t}\right)$ for a given measured sequence $A_{m}$ and set of $n$ marker trajectories $\left(p_{m 1}\right.$, $\left.p_{m 2}, \ldots, p_{m n}\right)$.

weights of the three unitless components $f_{\mathrm{PS}}, f_{\mathrm{RoM}}$, and $f_{d}$. The first component locates the direction of the PS axis with small variation in $\theta_{2}$ values, the second component serves to distinguish between FE and AA directions according to the RoM ratio, and the third component selects models with appropriate separation distances.

\section{Computation of Joint Parameters}

In our optimization approach, we search the domain of candidate joint models for the joint model with minimum cost according to the anatomy-based cost metric in (7). In this section, we present how to formulate the optimization domain as the 4-DOF set $\left(z_{h}, z_{t}\right)$ specifying the orientations of the FE and AA axes. Then, given a measured data sequence and a candidate set $\left(z_{h}, z_{t}\right)$, we can directly compute the remaining kinematic parameters and joint variables that are needed to evaluate the value of the cost metric. Pseudocode for the entire cost metric computation can be found in Fig. 4.

1) Kinematic Model: At any time sample $k$, the measured CMC joint configuration $A_{m}(k)$ is related to the functional joint configuration $A_{j}(k)$ by the two change-of-coordinate transforms [Fig. 3(a)]

$$
A_{m}(k)=A_{h} A_{j}(k) A_{t}
$$

where $A_{m}(k)$ is the measured configuration of the thumb metacarpal TCS with respect to the hand TCS at time $k, A_{h}$ is the fixed transform of the hand FCS in the hand TCS, $A_{j}(k)$ is the functional joint configuration of the metacarpal FCS in the hand FCS at time $k$, and $A_{t}$ is the fixed transform of the thumb
TABLE I

DH PARAMETERS FOR DESCRIBING THE FUNCTIONAL CONFIGURATION OF THE CMC JOINT MODEL

\begin{tabular}{cccccc}
\hline $\begin{array}{c}\text { link } \\
\text { number }\end{array}$ & $\begin{array}{c}\text { joint } \\
\text { axis }\end{array}$ & $\begin{array}{c}\text { joint angle } \\
\theta\end{array}$ & $\begin{array}{c}\text { link length } \\
a\end{array}$ & $\begin{array}{c}\text { link twist } \\
\alpha\end{array}$ & $\begin{array}{c}\text { joint offset } \\
\text { ( }\end{array}$ \\
\hline 1 & FE & $\theta_{1}$ & 0 & $-\frac{\pi}{2}$ & 0 \\
2 & PS & $\theta_{2}$ & 0 & $\frac{\pi}{2}$ & $d$ \\
3 & AA & $\theta_{3}$ & 0 & 0 & 0 \\
\hline
\end{tabular}

There are three rotational axes with no translational degrees of freedom Successive axes are mutually orthogonal, as indicated by the link twist values. The FE and AA axes are separated by joint offset $d$ along the PS axis that is the mutual perpendicular. The skew between the FE and AA axes is defined by the rotation angle $\theta_{2}$ about the PS axis.

metacarpal TCS in the thumb metacarpal FCS. The relation in (8) can be expanded in terms of the each transform's orientation component $R$ and location component $p$ :

$$
\begin{aligned}
A_{m}(k) & =\left[\begin{array}{cc}
R_{h} & p_{h} \\
\overrightarrow{0} & 1
\end{array}\right]\left[\begin{array}{cc}
R_{j}(k) & p_{j}(k) \\
\overrightarrow{0} & 1
\end{array}\right]\left[\begin{array}{cc}
R_{t} & p_{t} \\
\overrightarrow{0} & 1
\end{array}\right] \\
& =\left[\begin{array}{cc}
R_{m}(k) & p_{m}(k) \\
\overrightarrow{0} & 1
\end{array}\right] .
\end{aligned}
$$

We use the robotics Denavit-Hartenberg (DH) convention [18] to parameterize $A_{j}(k)$ for the kinematic model depicted in Fig. 3(b) (Table I). This convention provides a framework to describe both the joint axis orientation and joint axis location, and it has been used previously to describe the nonorthogonal and nonintersecting axes of the thumb joints in recent literature [4], [11]. The FE axis $z_{h}$ fixed in the hand frame is orthogonal to and intersects the PS axis, and the PS axis is orthogonal to and intersects the AA axis $z_{t}$ fixed in the thumb metacarpal frame. The distance between the intersection points on the PS axis defines the separation $d$ between the FE and AA axes, and the value of the PS rotation angle determines the skew angle between the FE and AA axes. The value for the PS rotation angle for nonsingular configurations will be near $\pi / 2$. The general form of $A_{j}(k)$ is expressed in terms of the fixed separation $d$ and the three joint angles $\theta_{1}, \theta_{2}$, and $\theta_{3}$, using the abbreviated notation $s_{i}=\cos \theta_{i}$ and $c_{i}=\sin \theta_{i}$, as

$$
A_{j}(k)=\left[\begin{array}{cccc}
c_{1} c_{2} c_{3}-s_{1} s_{3} & -c_{1} c_{2} s_{3}-s_{1} c_{3} & c_{1} s_{2} & -d s_{1} \\
s_{1} c_{2} c_{3}+c_{1} s_{3} & -s_{1} c_{2} s_{3}+c_{1} c_{3} & s_{1} s_{2} & d c_{1} \\
-s_{2} c_{3} & s_{2} s_{3} & c_{2} & 0 \\
0 & 0 & 0 & 1
\end{array}\right] .
$$

Overall, 13 fixed parameters specifying $A_{h}, A_{t}$, and $d$ describe the CMC joint model, while the values of the three joint variables at all $N$ time samples describe a measured motion sequence. Instead of optimizing over the high-DOF space $\left(A_{h}, A_{t}, d\right)$ and solving for the joint angles to compute the cost metric, we can simplify the search to a four-parameter domain for $\left(z_{h}, z_{t}\right)$ by using the measured motion $A_{m}$ to solve for the remaining nine parameters and $3 N$ joint variables. 
2) Joint Coordinate System Orientation: We first consider the orientation component of the model, because the orientation $R_{m}$ is independent of all location parameters, as seen from (9). The orientations of the joint axes are defined by only four numbers specifying the pair of axis directions $\left(z_{h}, z_{t}\right)$. The FE axis $z_{h}$ is the $z$-axis of $R_{h}$, the AA rotation axis $z_{t}$ is the $z$-axis of $R_{t}^{\mathrm{T}}$, and the PS axis is orthogonal to both $z_{h}$ and $z_{t}$. For each pair $\left(z_{h}, z_{t}\right)$, there is a family of solutions for the 6 DOF set $\left(R_{h}, R_{t}\right)$, where the corresponding functional joint angle sequences $\left(\theta_{1}, \theta_{2}, \theta_{3}\right)$ for a sequence of measured rotations $R_{m}(k)$ are fully defined up to a shift in the reference angle for $\theta_{1}$ and $\theta_{3}$ and a sign change for $\theta_{2}$. The ambiguity in the sign of $\theta_{2}$ is due to the fact that there are two sets of joints angles, $\left(\theta_{1}, \theta_{2}, \theta_{3}\right)$ and $\left(\theta_{1}+\pi,-\theta_{2}, \theta_{3}+\pi\right)$, corresponding to any single matrix $R_{j}(k)$.

We choose a canonical set of joint angle values from the family of solutions corresponding to a pair $\left(z_{h}, z_{t}\right)$ by selecting positive values for $\theta_{2}$ and reference configuration angles for $\theta_{1}$ and $\theta_{3}$ such that the modes of the observed angle sequences are zero (Fig. 4)

$$
\begin{aligned}
\operatorname{mode}\left(\theta_{1}\right) & =0 \\
\theta_{2} & >0 \\
\operatorname{mode}\left(\theta_{3}\right) & =0 .
\end{aligned}
$$

The mode is computed by mapping the $N$ continuous angle values to a discrete set of $5^{\circ}$ intervals and selecting the center value of the interval with the maximum frequency. In effect, (11) and (13) specify the remaining 2 DOFs that select a single 6-DOF set $\left(R_{h}, R_{t}\right)$ from a 4-DOF family defined by $\left(z_{h}, z_{t}\right)$. The appropriate set $\left(R_{h}, R_{t}\right)$ is computed by first estimating the joint angle sequences from an arbitrary choice of $\left(R_{h}, R_{t}\right)$ within the $\left(z_{h}, z_{t}\right)$ family and then shifting the reference configuration angles by the modes of the estimated sequences (Fig. 4, lines 1-14). Using the mode of the joint angle sequence approximately centers the angle values around zero and avoids angle values near $\pm \pi$. Typically, the positive values of $\theta_{2}$ selected by (12) will be near $+\pi / 2$.

Thus, for any candidate set of axis directions $\left(z_{h}, z_{t}\right)$ and a sequence of measured rotations $R_{m}(k)$, we can compute a canonical 6-DOF orientation pair $\left(R_{h}, R_{t}\right)$ and the set of three joint variable sequences $\left(\theta_{1}, \theta_{2}, \theta_{3}\right)$. The first part of Fig. 4 reviews this computation in pseudocode form.

3) Joint Axes Location Parameters: The locations of the joint axes are defined by two position vectors $p_{h}$ and $p_{t}$ (9), as well as the separation distance $d$. The position vector of the measured transform $A_{m}$ is a linear function of these location parameters, as derived from (9):

$$
p_{m}(k)=p_{h}+R_{h} u_{1}(k) d+R_{h} R_{j}(k) p_{t}
$$

where

$$
u_{1}(k)=\left[\begin{array}{c}
-s_{1}(k) \\
c_{1}(k) \\
0
\end{array}\right] \text {. }
$$

Given a candidate set of orientations $\left(R_{h}, R_{t}\right)$ that define the joint angle values, we can solve directly for the location values $\left(p_{h}, p_{t}, d\right)$ that minimize marker reconstruction error as a linear least squares problem. Each time sample $k$ provides the following set of equations for the positions $\left(p_{m 1}, p_{m 2}, p_{m 3}\right)$ of the three markers on the metacarpal segment

$$
\begin{aligned}
& {\left[\begin{array}{l}
p_{m 1}(k) \\
p_{m 2}(k) \\
p_{m 3}(k)
\end{array}\right]=} \\
& {\left[\begin{array}{ccccc}
I_{3} & R_{h} u_{1} & R_{h} R_{j}(k) & 0_{3} & 0_{3} \\
I_{3} & R_{h} u_{1} & 0_{3} & R_{h} R_{j}(k) & 0_{3} \\
I_{3} & R_{h} u_{1} & 0_{3} & 0_{3} & R_{h} R_{j}(k)
\end{array}\right]\left[\begin{array}{c}
p_{h} \\
d \\
p_{t 1} \\
p_{t 2} \\
p_{t 3}
\end{array}\right]}
\end{aligned}
$$

where $I_{3}$ is the $3 \times 3$ identity matrix and $0_{3}$ is a $3 \times 3$ matrix of zeros. The measurements from all $N$ time samples are combined in an overdetermined system of equations that is solved in a least squares manner for the $13 \times 1$ location parameter vector $\left(p_{h}, d, p_{t 1}, p_{t 2}, p_{t 3}\right)$. This completes the full specification of $\left(A_{h}, A_{t}\right)$ defining the orientation and location of the CMC joint axes, and in addition, provides the separation distance $d$ between the FE and AA axes.

Conceptually, the axis orientations $\left(z_{h}, z_{t}\right)$ are defined by two bidirectional lines, but in practice, the parameterization using directed vectors results in multiple equivalent solutions in the 4-DOF space that differ by a sign change. For a physical interpretation of the separation distance $d$, we denote $d \geq 0$ when the $\mathrm{AA}$ axis is distal to the $\mathrm{FE}$ axis and $d<0$ when the AA axis is proximal to the FE axis. The middle section of Fig. 4 (lines 15-19) reviews this calculation in pseudocode form.

\section{E. Data Analysis}

We determined individual CMC joint models from the experimental data by optimizing the cost metric defined in (7), whose three components model prior anatomic knowledge of the CMC joint. The tuning parameters were set to $\sigma_{2}=5^{\circ}, \mu_{d}=5 \mathrm{~mm}$, and $\sigma_{d}=5 \mathrm{~mm}$. The small value of $\sigma_{2}$ corresponds to small amounts of PS rotation. The positive value of $\mu_{d}$ reflects the anatomic joint property that the AA axis is distal to the FE axis, and the value of $\sigma_{d}$ indicates the expected variation in the anatomically-plausible separation distances. With these values, the cost metric components are of approximately the same magnitude, as determined from sensitivity tests of the parameter values.

We also tested three other competing approaches for determining the axis orientations of an individual CMC joint. First, we consider a constant set of in vitro axis orientations that is applied to all individuals. Cooney et al. [5] measured the CMC axes based on in vitro bone surface geometry for ten cadavers and reported the mean orientations of the trapezium axes with respect to the hand dorsum coordinate frame. We converted the reported results from Cooney et al. [5] for a fixed-axis rotation 
convention, where joint axes are fixed in the trapezium frame, to a moving-axis rotation convention for comparison to our model, where the AA axis is fixed in the metacarpal frame and moves relative to the trapezium. The joint coordinate system reported by Cooney et al. [5] only defines the axis orientation but not the axis locations. We will calculate the location parameters for each individual using the joint angles corresponding to the set of constant axis directions $\left(z_{h}, z_{t}\right)$ converted from the results of Cooney et al. [5] (further described later).

Second, we test an optimization approach whose minimization cost metric is the marker trajectory reconstruction error for a 3-DOF joint model. For a candidate set of joint axis directions $\left(z_{h}, z_{t}\right)$, the full set of three joint angles and the location parameters were computed as described previously for our method. The cost for the set $\left(z_{h}, z_{t}\right)$ is then calculated from the joint angles and location parameters as the rms distance between the measured and predicted marker positions over all the markers and all time samples.

The third approach we tested is an optimization of the marker trajectory reconstruction error for a 2-DOF joint model. We use this model to investigate how model constraints affect the optimization of reconstruction error. To perform this optimization, we computed reconstruction error as follows. After solving for the full set of three joint angles and location parameters given a pair $\left(z_{h}, z_{t}\right)$, the value of $\theta_{2}$ was fixed to the mean PS joint angle. The marker reconstruction error for a 2-DOF model was then computed from the time-varying joint angle values of $\theta_{1}$ and $\theta_{3}$, the constant value of $\theta_{2}$, and the location parameters.

The optimization method used for the three optimization approaches was implemented in MATLAB (R2006a, Mathworks, Inc., Natick, MA) using the built-in simplex optimization algorithm fminsearch to minimize the cost metric. The fourdimensional search domain represents the set of axis directions $\left(z_{h}, z_{t}\right)$. The two numbers for each $z$-axis direction are the $x$ and $y$ components of an axis-angle rotation that aligns the current $z$-axis to the new $z$-axis direction. To improve the quality of the solution and address the problem of local minima, the search was initialized ten times. For each initialization, the two axis-angle rotations defining the directions $\left(z_{h}, z_{t}\right)$ were chosen randomly from a uniform distribution of $(x, y)$ points within a circle of radius $\pi / 2$. This distribution corresponds to one hemisphere of possible $z$-axis distributions. The best overall local minimum from the ten initializations was selected as the final solution for the given data sequence. Preliminary testing on a small sample of the available data determined the choice of ten initializations to be sufficient for repeatable optimization results.

For all three optimization approaches, the optimization technique was applied separately to the two repetitions of the calibration movement. The two resulting joint models were compared in twofold cross-validation where the cost metric was evaluated on the motion of one repetition using the optimization result for $\left(z_{h}, z_{t}\right)$ from the other repetition. The solution with the lower cross-validation cost was selected as the final single CMC model for the particular subject hand.

For each of the four approaches, the solution for $\left(z_{h}, z_{t}\right)$ was then used to compute the corresponding joint variable values and the location parameters from the combined data set of both calibration movement repetitions. The average skew between the FE and AA axes is the mean value of the PS angle $\theta_{2}$ from the recorded movement. RoM for all three joint angles was measured as the difference between the maximum and minimum values, as in (5). The rms marker position reconstruction error can be computed from the estimated location parameters and joint angle values. Note that, for the marker reconstruction optimization approach of the 2-DOF model, the value of $\theta_{2}$ is only fixed to the mean joint angle for computing the cost metric, but for reporting results, the full set of varying values for $\theta_{1}, \theta_{2}$, and $\theta_{3}$ is used for evaluation of the RoM, location parameters, and marker reconstruction error.

For the three optimization approaches, the mean direction of the axes $z_{h}$ and $z_{t}$ was calculated by representing each subjectspecific direction as a point on the unit sphere and using the spherical averaging technique developed by Buss and Fillmore [19]. The results for the left hand were converted to the right hand coordinate frame, such that a single distribution of axis orientations included results for both hands of all subjects. The mean direction $\bar{z}$ was compared to the constant in vitro joint axis direction $z_{c}$ reported by Cooney et al. [5]. Intersubject variability is measured from the angular deviation of an individual axis orientation $z$ relative to the mean axis orientation $\bar{z}$.

\section{RESULTS}

Optimizing the anatomy-based cost metric resulted in an intuitive alignment of the $\mathrm{CMC}$ axes due to the qualitative characteristics modeled in the cost metric (Fig. 5). The average axis directions from our proposed optimization method differed from the constant in vitro axis directions by $20^{\circ}$ and $35^{\circ}$ for $z_{h}$ and $z_{t}$, respectively (Table II). The deviation of a subjectspecific axis to the mean axis was at most $27^{\circ}$ and $53^{\circ}$ for $z_{h}$ and $z_{t}$. In contrast, the optimization of marker reconstruction error for both the 3-DOF and 2-DOF joint models failed to consistently estimate anatomically plausible directions. For the reconstruction optimization of a 3-DOF model, the deviation of a subject-specific axis was as much as $105^{\circ}$ and $96^{\circ}$ for $z_{h}$ and $z_{t}$, respectively, indicating a lack of consistent axis directions. The reconstruction optimization of the 2-DOF model also resulted in large maximum deviations of $105^{\circ}$ and $95^{\circ}$ for $z_{h}$ and $z_{t}$, respectively, and the average location of the AA axis in the metacarpal frame differed unacceptably from $z_{c}$ by $72^{\circ}$.

For all four approaches, the resulting RoMs (Table III) decrease in the order of FE, AA, and PS movement, as in previous descriptions of the functional CMC motion [1]. However, the RoMs determined from our anatomy-based optimization were the most consistent with the concept that the CMC joint is predominantly a 2-DOF joint. The PS RoM was on average $23^{\circ}$ and at most $34^{\circ}$ using our approach, while for the other three approaches, the average was at least $28^{\circ}$ and the maximum was at least $49^{\circ}$. In addition, the anatomy-based optimization measured overall larger FE RoM than the other two models due to the alignment of the axis orientations to each subject's specific movement. In a comparison of the marker reconstruction optimization for a 3-DOF model and a 2-DOF model, the constraint in the 2-DOF model results in a smaller PS RoM. The 
(a)
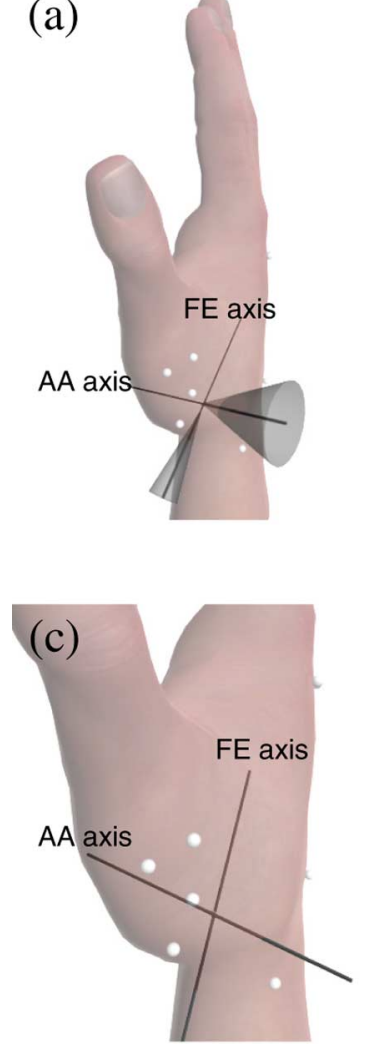

(b)
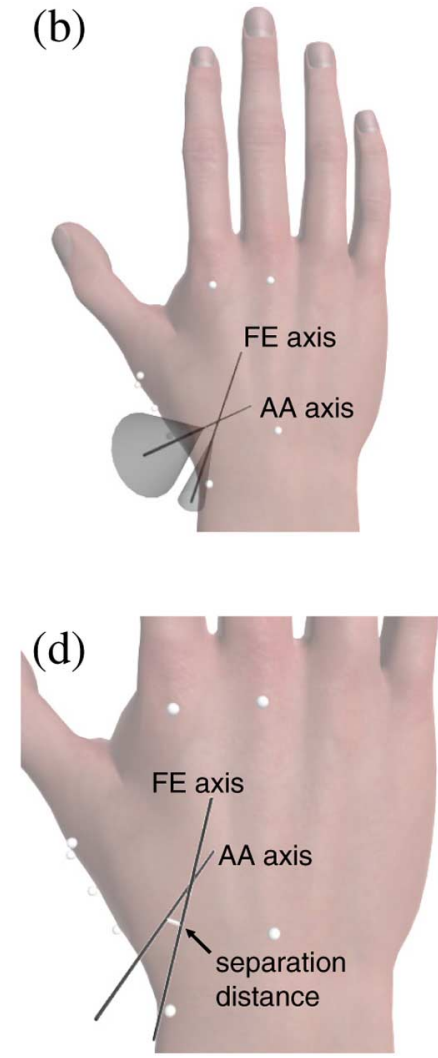

Fig. 5. (a) and (b) CMC joint model results for our anatomy-based optimization approach are represented by average directions of the FE axis and AA axis over 48 hands from 24 subjects. The cone denotes the mean angular deviation from the mean direction (Table II). (c) and (d) Close-up views of the CMC joint model for one example subject. (c) In the radial view, the separation distance between joint axes is nearly orthogonal to the page. (d) In the dorsal view, the white line highlights the separation distance between the FE and AA axis locations.

optimization of marker reconstruction error for the 3-DOF model is the least constrained with respect to possible joint angle values. Due to the fewer constraints, the optimization result with the least reconstruction error occurred near the rotation singularities $\theta_{2}=\pi$ such that the FE RoM and AA RoM were greater than $145^{\circ}$ for two individual thumbs.

The anatomy-based optimization also resulted in models where the FE axis was always proximal to the AA axis, unlike the other models that resulted in the FE axis distal to the AA axis for $10 \%, 35 \%$, and $67 \%$, respectively, of the thumbs for the constant axis orientation model, marker reconstruction optimization of the 3-DOF model, and marker reconstruction optimization of the 2-DOF model. Overall, for our anatomybased approach, the separation distance between the FE and $\mathrm{AA}$ axes of each individual $\mathrm{CMC}$ joint model was $4.6 \mathrm{~mm}$ on average, with $1.2 \mathrm{~mm}$ standard deviation across all hands. The mean value of the PS angle $\theta_{2}$, representing skew between the $\mathrm{FE}$ and $\mathrm{AA}$ axes, was $87 \pm 17^{\circ}$ (mean \pm standard deviation) from the positive flexion axis to the positive abduction axis. Average rms reconstruction error for the calibration movement was $2.4 \mathrm{~mm}$ per marker, which was only slightly greater than 2.2 , 1.9 , and $2.0 \mathrm{~mm}$ for the constant axis orientation model, marker reconstruction optimization of the 3-DOF joint model, and marker reconstruction optimization of the 2-DOF joint model, respectively.

Repeatability of the methods is measured by the difference between the optimized parameter values for the two separate repetitions of the calibration movement (Table IV). By definition, the constant axis orientation model is $100 \%$ repeatable for the axis orientations since it models zero inter- and intrasubject variability. In comparing the three optimization approaches, the resulting FE axis direction with maximum RoM estimated from our anatomy-based approach was repeatable for at least an additional $31 \%$ of the individual hands than that from either of the two marker reconstruction optimizations. The difference in repeatability of the AA axis with medium RoM was less clear with respect to the reconstruction approach for the 2-DOF model. This suggests that the FE axis with maximum RoM is more well defined than the AA axis with medium RoM. For the relative configurations of the FE and AA axes, our anatomy-based approach had the maximum repeatability of all three approaches for values of the mean $\theta_{2}$ skew angle within $1^{\circ}$ and the separation distance $d$ within $0.5 \mathrm{~mm}$. Overall, our optimization of the anatomy-based cost metric allowed for intersubject variation of the joint axis directions and in addition resulted in improved intrasubject repeatability of the separation distance and skew angle between the dominant axes.

\section{DISCUSSION}

Our method fits a subject-specific CMC joint model for evaluating $\mathrm{CMC}$ mobility with respect to axes that are aligned to an individual's particular motion. The anatomy-based cost metric reflects the preference for consistency with known CMC characteristics over purely minimizing the marker reconstruction error, which may result in anatomically-implausible parameter estimates. A convenient feature of the method is the simplified parameterization of the problem such that the pair of axis orientations $\left(z_{h}, z_{t}\right)$ are sufficient to determine the entire joint model, including axis locations, separation distance between axes, and skew angle between axes. The low-dimensional parameterization of candidate joint models simplifies the complexity of the search space that reduces the computational expense of the optimization, and a reasonable optimum is found with a small number of reinitializations.

\section{A. Description of Thumb Kinematics}

Measurements of the functional joint angles across 48 hands from 24 subjects show that CMC mobility can be described by two dominant DOFs of FE rotation and AA rotation. However, the amount of PS rotation is not necessarily negligible given that the PS RoM for active movement was $23^{\circ}$ on average and as high as $34^{\circ}$ for one individual. Investigation of the joint angle trajectories for a subset of the measured sequences did not find that any specific part of the calibration motion consistently exhibited greater PS rotation. PS movement was present throughout the calibration movement, and the PS RoM differed by only a few degrees between the four parts. Our technique provides a way to determine the two dominant axes of rotation without precluding measurement of the PS RoM, as would be necessary for 
TABLE II

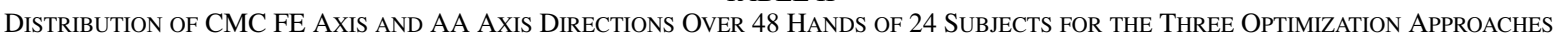

\begin{tabular}{|c|c|c|c|c|c|}
\hline \multirow{6}{*}{$z_{1}^{z_{c}}$} & \multirow{4}{*}{$\begin{array}{l}\text { Model } \\
\text { Marker reconstruction optimization, 3-DoF model }\end{array}$} & \multicolumn{4}{|c|}{ Angular deviation (degrees) from mean axis $\bar{z}$ to } \\
\hline & & \multicolumn{2}{|c|}{$\begin{array}{l}\text { Constant axis } z_{c} \text { from } \\
\text { in vitro joint model, } \gamma\end{array}$} & \multicolumn{2}{|c|}{$\begin{array}{l}\text { Subject-specific axis } z \\
\text { mean, } \delta[\text { maximum, } \Delta]\end{array}$} \\
\hline & & $z_{h}(\mathrm{FE})$ & $z_{t}(\mathrm{AA})$ & $z_{h}(\mathrm{FE})$ & $z_{t}(\mathrm{AA})$ \\
\hline & & 26 & 7 & $44[105]$ & $45[96]$ \\
\hline & Marker reconstruction optimization, 2-DoF model & 40 & 72 & $38[105]$ & $38[95$ \\
\hline & Anatomy-based optimization (our method) & 20 & 34 & $9\left[\begin{array}{ll}27 & 27\end{array}\right.$ & $22[53]$ \\
\hline
\end{tabular}

We compare the mean direction from each set of optimized axes to fixed axis orientations determined from in vitro bone surface geometry by Cooney et al. [5]. The mean and maximum deviation of the optimized axes reflect the amount of intersubject variability corresponding to each model.

TABLE III

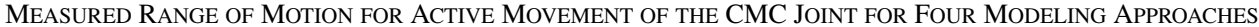

\begin{tabular}{|c|c|c|c|}
\hline \multirow[t]{2}{*}{ Model } & \multicolumn{3}{|c|}{$\begin{array}{c}\text { Active range of motion (degrees) } \\
\text { mean [ maximum ] }\end{array}$} \\
\hline & $\theta_{1}(\mathrm{FE})$ & $\theta_{2}(\mathrm{PS})$ & $\theta_{3}(\mathrm{AA})$ \\
\hline Constant axis orientation model & $69[87]$ & 29 [49] & 46 [ 77] \\
\hline Marker reconstruction optimization, 3-DoF model & $66[182]$ & $44[71]$ & $62[164]$ \\
\hline Marker reconstruction optimization, 2-DoF model & 71 [146] & [51] & [112] \\
\hline Anatomy-based optimization (our method) & 76 [ 98] & 23 [34] & 43 [ 69] \\
\hline
\end{tabular}

previous models that assume a priori that the joint has only two rotational DOFs.

Our noninvasive technique fits a subject-specific model to motion data that samples the entire space of in vivo $\mathrm{CMC}$ joint configurations, rather than determining the axis orientations from simplified movements restricted to, for example, only flexion or circumduction. We found that the AA axis was less well defined than the FE axis, and future studies are needed to explore whether this is explained by the anatomical joint constraints. In addition, our method can measure the average skew angle and separation distance between the FE axis and AA axis, and our estimates for both values were anatomically plausible.

\section{B. Crafting a Reliable Cost Metric}

Our choice of optimization cost metric components is based on anatomic $\mathrm{CMC}$ joint properties, rather than a minimization of only the marker trajectory reconstruction error as used in prior work [14]-[16]. Optimizing the reconstructed marker positions was insufficient for fitting an anatomically-meaningful model, since there may be several candidate solutions with low reconstruction error but whose corresponding joint angle values are inconsistent with functional anatomical descriptions. In addition, using the reconstruction error as the cost metric resulted in decreased repeatability of the estimated kinematic parameters (Table IV). The loss in the anatomical plausibility and repeatability corresponds to only a slight improvement in the mean rms reconstruction error from $2.4 \mathrm{~mm}$ for our method to $1.9 \mathrm{~mm}$ in the best alternative we tested. It is possible that the small scale of the hand complicates the applicability of evaluating only marker reconstruction error to fit anatomically-meaningful joint axes, which has been successfully accomplished for other joints of the body [14]-[16].

Our anatomy-based approach allowed us to incorporate prior knowledge about the CMC joint to solve for anatomicallyplausible joint parameters, and the results of our method do depend on the selected values of the weighting parameters used to tune the cost metric. Adjusting the weights, and thus, relative magnitudes, of the cost components will bias the result toward joint models that satisfy the modeled constraints to different degrees. Our experience was that, even in the reduced dimensionality of the search space, there may be several near-optimum solutions associated with each individual cost component such that an unbalanced relative weighting may not satisfy all of the constraints reasonably. For example, choosing $\sigma_{2}=10^{\circ}$ in our sensitivity tests relaxes the constraint for small variation in the PS angle that for some subjects, led to a solution where the AA and PS axes were misaligned to achieve a smaller value of $f_{\text {RoM }}$. The parameters of $\mu_{d}$ and $\sigma_{d}$ were also critical for weighting the cost metric toward positive values of the separation distance without compromising the metrics on the joint angles. Selecting $\mu_{d}=10 \mathrm{~mm}$ and $\sigma_{d}=5 \mathrm{~mm}$, for example, did result in larger separation distance values, but the axis orientations were misaligned for a few subjects such that the AA RoM was greater than the FE RoM or the PS RoM was greater than the AA RoM. Given the importance of selecting appropriate weights for the optimization, the sensitivity to optimization parameters might be further investigated using Monte Carlo simulations to determine the distribution of possible parameter values. In addition, 
TABLE IV

Repeatability of Kinematic Parameter Estimates Based on the Difference Between Parameter Values Estimated From Two SEPARATE REPETITIONS OF THE ACTIVE ROM CALIBRATION MOVEMENT

\begin{tabular}{|c|c|c|c|c|}
\hline \multirow{2}{*}{$\begin{array}{l}\text { Estimated parameter } \\
\text { Orientation parameters }\end{array}$} & \multirow[t]{2}{*}{ Model } & \multicolumn{3}{|c|}{$\begin{array}{l}\text { Percent of hands where difference be- } \\
\text { tween two estimates is within }\end{array}$} \\
\hline & & 5 degrees & 10 degrees & 20 degrees \\
\hline \multirow[t]{4}{*}{$z_{h}$ (FE axis) direction } & Constant axis orientation model & 100 & 100 & 100 \\
\hline & Marker reconstruction optimization, 3-DoF model & 8 & 25 & 38 \\
\hline & Marker reconstruction optimization, 2-DoF model & 33 & 58 & 69 \\
\hline & Anatomy-based optimization (our method) & 65 & 94 & 100 \\
\hline \multirow[t]{5}{*}{$z_{t}$ (AA axis) direction } & Constant axis orientation model & 100 & 100 & 100 \\
\hline & Marker reconstruction optimization, 3-DoF model & 17 & 27 & 46 \\
\hline & Marker reconstruction optimization, 2-DoF model & 31 & 52 & 67 \\
\hline & Anatomy-based optimization (our method) & 23 & 54 & 88 \\
\hline & & 1 degree & 5 degrees & 10 degrees \\
\hline \multirow{4}{*}{$\begin{array}{l}\text { Mean } \theta_{2} \text { value, } \\
\text { defining skew between } \\
\text { FE and AA axes }\end{array}$} & Constant axis orientation model & 35 & 60 & 92 \\
\hline & Marker reconstruction optimization, 3-DoF model & 4 & 23 & 40 \\
\hline & Marker reconstruction optimization, 2-DoF model & 27 & 44 & 60 \\
\hline & Anatomy-based optimization (our method) & 77 & 88 & 98 \\
\hline Location parameter & & $0.5 \mathrm{~mm}$ & $1 \mathrm{~mm}$ & $2 \mathrm{~mm}$ \\
\hline \multirow{4}{*}{$\begin{array}{l}\text { Separation } d \text { between } \\
\text { FE and AA axes }\end{array}$} & Constant axis orientation model & 60 & 98 & 100 \\
\hline & Marker reconstruction optimization, 3-DoF model & 23 & 52 & 65 \\
\hline & Marker reconstruction optimization, 2-DoF model & 44 & 73 & 81 \\
\hline & Anatomy-based optimization (our method) & 88 & 100 & 100 \\
\hline
\end{tabular}

The results of using our anatomy-based cost metric are compared to results obtained from the constant axis orientation model reported by Cooney et al. [5] and two subject-specific optimization approaches that minimize marker trajectory reconstruction error.

the weighting parameters in this study reflect prior knowledge of unimpaired CMC joints, and more work is needed to determine how to change the parameters and cost metric for evaluation of pathological joints.

\section{Marker Protocol Considerations}

Although our experiments used the same marker protocol for all subjects for the purpose of reporting intersubject variability with respect to a consistent reference frame, the presented optimization framework is general and can work with any arbitrary choice of the hand TCS and thumb TCS that define the configurations of trapezium bone and thumb metacarpal bone. When using surface marker techniques, however, the marker placement should still be designed carefully to minimize systematic error due to the difference between the TCS on the skin surface and the ideal coordinate system of the bone. The quality of the optimization result is also limited by the amount of nonrigidity of the marker set.

For our experimental protocol, relative motion between the trapezium bone and the metacarpal bones or between the second and third metacarpal bones are potential sources of systematic artifacts in the TCS measurement. Skin movement relative to the bone also affects the reliability of the TCS measurement. We tried to reduce these artifacts by spacing out marker positions over the segments and directing subjects to avoid motion of other hand joints during the calibration movement. The motion artifacts may be interpreted as additional rotation or translation of the coordinate frames, but our results suggest that the optimization method finds anatomically plausible orientations of the joint axes that can be used to reasonably evaluate the joint RoM. Further investigation is required to determine the robustness to additional skin artifacts resulting from more functionallyrelevant calibration movements that include motion of the other hand joints.

Another point of caution is that the cost metric component modeling the variance of the PS angle and the least squares estimate of the location parameters are sensitive to outliers in the marker trajectories. The perturbation due to outliers was reduced by omitting any time samples with occluded markers rather than manually filling the trajectory gaps. This was possible since our method requires only a set of multiple CMC joint configurations that is not necessarily a continuous motion trajectory. In addition, the least squares estimate of the location parameters uses the positions of multiple markers on the thumb metacarpal instead of only the single marker denoting the thumb 
TCS frame origin. While this does not account for systematic error affecting the entire marker set, we are able to estimate the parameters describing the location and separation between joint axes that are consistent with the CMC joint anatomy even in the presence of skin deformation.

\section{Extensions for Further Study}

Although we use one specific DH parameterization of the motion transform $A_{j}$, the method can be adapted for other representations of three sequential rotations about the coordinate system axes. In all cases, the orientation of the joint axes are independent of the position parameters of the joint model. Regardless of the order of coordinate axes rotation used to parameterize $R_{j}$, one column vector of $R_{h}$ denotes the first axis of rotation and one vector in $R_{t}^{\Gamma}$ denotes the third axis of rotation. Thus, the reduced 4 DOF parameterization for the joint axes can be used with other rotation conventions.

Our method solves for a set of joint axes that are aligned to an individual's pattern of motion, and it is suitable for objectively and noninvasively measuring the $\mathrm{CMC}$ joint mobility from the three joint angle RoMs. In addition, the method can be used in a skeletal-fitting procedure to automatically construct a subjectspecific kinematic hand model for applications, such as haptic interfaces, virtual rehabilitation systems, and computer graphics. The developed framework is not limited to surface marker techniques, as it can accommodate any experimental technology that measures the relative transform between a TCS defining the trapezium frame and a TCS defining the thumb metacarpal bone frame. A potential avenue for further research is to use the optimization method together with medical imaging techniques that measure the bone configurations directly to investigate the relationship between the axes derived from the motion pattern and the axes defined by bone surface geometry. The estimation of the joint axes orientation and location parameters may then be used for periodic evaluation of the bone surface wear, soft tissue deterioration, and changes in thumb mobility.

\section{ACKNOWLEDGMENT}

The authors thank J. Macey for their assistance with the data acquisition and M. Mahler for creating the hand models. Autodesk provided the Maya software used for rendering the hand model images.

\section{REFERENCES}

[1] I. A. Kapandji, The Physiology of the Joints. 2nd ed. Edinburgh: E\&S Livingstone, 1970, vol. 1.

[2] T. Imaeda, K.-N. An, and W. P. Cooney, "Functional anatomy and biomechanics of the thumb," Hand Clin., vol. 8, no. 1, pp. 9-15, 1992.

[3] A. Hollister, W. L. Buford, L. M. Myers, D. J. Giurintano, and A. Novick, "The axes of rotation of the thumb carpometacarpal joint," J. Orthop. Res., vol. 10, no. 3, pp. 454-460, May. 1992.

[4] V. J. Santos and F. J. Valero Cuevas, "Reported anatomical variability naturally leads to multimodal distributions of Denavit-Hartenberg parameters for the human thumb," IEEE Trans. Biomed. Eng., vol. 53, no. 2, pp. 155-163, Feb. 2006.

[5] W. P. Cooney, M. J. Lucca, E. Y. Chao, and R. L. Linscheid, "The kinesiology of the thumb trapeziometacarpal joint," J. Bone Joint Surg. Amer., vol. 63, no. 9, pp. 1371-1381, Dec. 1981 .
[6] J. H. Coert, H. G. van Dijke, S. E. Hovius, C. J. Snijders, and M. F. Meek, "Quantifying thumb rotation during circumduction utilizing a video technique," Orthop. Res., vol. 21, no. 6, pp. 1151-1155, Nov. 2003.

[7] X. Zhang, P. Braido, S. W. Lee, R. Hefner, and M. Redden, "A normative database of thumb circumduction in vivo: Center of rotation and range of motion," Hum. Factors, vol. 47, no. 3, pp. 550-561, 2005.

[8] L. Chèze, N. Doriot, M. Eckert, C. Rumelhart, and J. J. Comtet, "In vivo cinematic study of the trapezometacarpal joint," Chir. Main., vol. 20, no. 1, pp. 23-30, Feb. 2001 (in French).

[9] P. Cerveri, E. D. Momi, N. Lopomo, G. Baud Bovy, P. Pajardi, and G. Ferrigno, "In-vivo estimation of the kinematic parameters of the trapezio-metacarpal joint using surface markers," J. Biomech., vol. 39, no. 1 , p. S82, 2006.

[10] F. J. Valero Cuevas, M. E. Johanson, and J. D. Towles, "Towards a realistic biomechanical model of the thumb: The choice of kinematic description may be more critical than the solution method or the variability/uncertainty of musculoskeletal parameters," J. Biomech., vol. 36, no. 7, pp. 10191030, Jul. 2003.

[11] D. J. Giurintano, A. M. Hollister, W. L. Buford, D. E. Thompson, and L. M. Myers, "A virtual five-link model of the thumb," Med. Eng. Phys., vol. 17, no. 4, pp. 297-303, Jun. 1995.

[12] J. A. Katarincic, "Thumb kinematics and their relevance to function," Hand Clin., vol. 17, no. 2, pp. 169-174, May. 2001.

[13] P. W. Brand and A. Hollister, Clinical Mechanics of the Hand. 3rd ed. St. Louis, MO: Mosby, 1993, pp. 121-122.

[14] H. J. Sommer and N. R. Miller, "A technique for kinematic modeling of anatomical joints," J. Biomech. Eng., vol. 102, no. 4, pp. 311-317, 1980.

[15] A. van den Bogert, G. D. Smith, and B. M. Nigg, "In vivo determination of the anatomical axes of the ankle joint complex: an optimization approach," J. Biomech., vol. 27, no. 12, pp. 1477-1488, 1994.

[16] J. A. Reinbolt, J. F. Schutte, B. J. Fregly, B. I. Koh, R. T. Haftka, A. D. George, and K. H. Mitchell, "Determination of patient-specific multi-joint kinematic models through two-level optimization," $J$. Biomech., vol. 38, no. 3, pp. 621-626, 2005.

[17] C. M. Bishop, Pattern Recognition and Machine Learning. New York, NY: Springer, 2006, pp. 221-28.

[18] M. W. Spong, S. Hutchinson, and M. Vidyasagar, Robot Modeling and Control. Hoboken, NJ: Wiley, 2006, ch. 3.2.

[19] S. R. Buss and J. P. Fillmore, "Spherical averages and applications to spherical splines and interpolation," ACM Trans. Graph., vol. 20, no. 2, pp. $95-126$, Apr. 2001

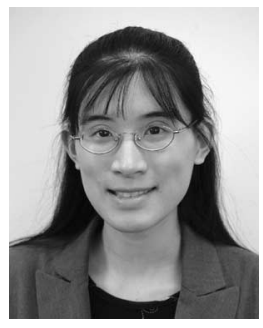

Lillian Y. Chang (S'06) received the M.S. degree in robotics in 2006 from Carnegie Mellon University, Pittsburgh, PA, where she is currently a Graduate Research Assistant at the Robotics Institute.

Her research interests include biomechanical modeling of the hand, dexterous manipulation in humans, and control of anthropomorphic robotic manipulators.

Ms. Chang was the recipient of the National Science Foundation Graduate Research Fellowship in 2004.

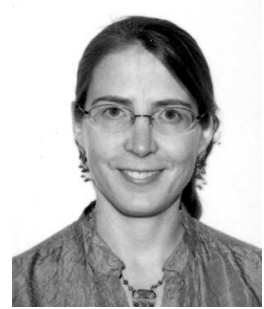

Nancy S. Pollard (S'88-M'98) received the Ph.D degree in electrical engineering and computer science from the Artificial Intelligence Laboratory, Massachusetts Institute of Technolgy (MIT), Cambridge, in 1994.

She was an Assistant Professor at Brown University, Providence, RI, where she was also part of the Computer Graphics Group. At MIT, she was engaged in research on grasp planning for articulated robot hands. She is currently an Associate Professor in the School of Computer Science, Robotics Institute, Carnegie Mellon University, Pittsburgh, PA.

Dr. Pollard was the recipient of the 2001 National Science Foundation (NSF) CAREER Award for research on "Quantifying Humanlike Enveloping Grasps" and the Okawa Research Grant in 2006 for "Studies of Dexterity for Computer Graphics and Robotics." 